\title{
PERTURBATION ANALYSIS FOR PALINDROMIC AND ANTI-PALINDROMIC NONLINEAR EIGENVALUE PROBLEMS*
}

\author{
SK. SAFIQUE AHMAD ${ }^{\dagger}$
}

\begin{abstract}
A structured backward error analysis for an approximate eigenpair of structured nonlinear matrix equations with $T$-palindromic, $H$-palindromic, $T$-anti-palindromic, and $H$-anti-palindromic structures is conducted We construct a minimal structured perturbation in the Frobenius norm such that an approximate eigenpair becomes an exact eigenpair of an appropriately perturbed nonlinear matrix equation. The present work shows that our general framework extends existing results in the literature on the perturbation theory of matrix polynomials.
\end{abstract}

Key words. nonlinear eigenvalue problem, even and odd matrix polynomials, palindromic matrix polynomial

AMS subject classifications. 65F15, 15A18, 65F35, 15A12

1. Introduction. We consider the perturbation analysis of eigenvalue problems of the form

$$
\left(\sum_{j=0}^{m} M_{j} f_{j}(c, s)\right) x=0,
$$

for some nonzero vector $x \in \mathbb{C}^{n}$. Here, $M_{j} \in \mathbb{C}^{n \times n}$, for $j=0,1, \ldots, m$, and $f_{j}(c, s)$ are scalar-valued functions with $(c, s) \in \mathbb{C}^{2} \backslash\{(0,0)\}$. The pair $(c, s)$ is called an eigenvalue of $\sum_{j=0}^{m} M_{j} f_{j}$, and $x$ is the corresponding eigenvector. For the sake of convenience, we write (1.1) as

$$
\begin{aligned}
(M \otimes f(c, s)) x & =\left(\sum_{j=0}^{m} M_{j} f_{j}(c, s)\right) x=0, \quad \text { where } \\
M & =\left(M_{0}, \ldots, M_{m}\right) \in\left(\mathbb{C}^{n \times n}\right)^{m+1} \quad \text { and } \\
f(c, s) & =\left(f_{0}(c, s), f_{1}(c, s), \ldots, f_{m}(c, s)\right) \in \mathbb{C}^{m+1} .
\end{aligned}
$$

The components $f_{j}(c, s)$ are rational functions, where $(c, s)$ is defined on the Riemann sphere $\mathcal{R}:=\left\{(c, s) \in \mathbb{C}^{2} \backslash\{(0,0)\}:|c|^{2}+|s|^{2}=1\right\}$. Even though the perturbation theory for a non-homogeneous setup for matrix polynomials is well investigated [2], no literature is available for nonlinear eigenvalue problems of the form (1.2). However, in recent years nonlinear eigenvalue problems, more specifically palindromic eigenvalue problems, have found application in various areas of science and engineering [16]. An illustrative example is the vibration of fast trains [16].

The vibration of fast trains leads to a palindromic quadratic eigenvalue problem which is given as $\left(\lambda^{2} A^{T}+\lambda C+A\right) x=0$, where $A, C=C^{T} \in \mathbb{C}^{n \times n}$. In general most of the eigenvalues of the quadratic eigenvalue problems are of the form

$$
\left(\lambda M_{1}(\omega)+M_{0}(\omega)+\frac{1}{\lambda} M_{1}(\omega)^{T}\right) v=0,
$$

where $M_{0}(\omega), M_{1}(\omega)$ are large and sparse complex matrices depending on $\omega$ such that $M_{1}(\omega)$ is highly rank deficient and $M_{0}(\omega)$ is complex symmetric; see $[6,16]$. The scarcity of work

*Received May 23, 2018. Accepted March 27, 2019. Published online on June 24, 2019. Recommended by Wen-Wei Lin. Research work funded by DST, India, Grant Number: SR/FTP/MS-043/2011.

${ }^{\dagger}$ Discipline of Mathematics, Indian Institute of Technology Indore, Simrol, Indore-453552, Madhya Pradesh, India (safiquediti.ac.in, safiquedgmail.com). 
on the nonlinear eigenvalue problem has motivated us to take up this study on the perturbation analysis for nonlinear matrix equations.

The present work deals with the palindromic rational eigenvalue problem in the homogeneous framework. The homogeneous framework helps to study the problem (1.2) in the presence of the eigenvalues 0 and $\infty$ in linear/nonlinear palindromic rational eigenvalue problems. Our framework can be applied as long as there is no pole of the rational functions $f_{j}(c, s)$ in equation (1.2). A nonlinear eigenvalue problem of the form (1.2) can be converted into a linear eigenvalue problem by the usual linearization approaches; see, e.g., [9, 10, 11, 16, 17]. However, we have not adopted this approach due to the presence of rounding errors. Bounds for the structured backward errors are given by Li et al. in [15] for non-homogeneous palindromic matrix polynomials. In this article, efforts have been made to introduce the exact formula for the backward error such that an approximate eigenpair becomes an exact eigenpair of an appropriately perturbed nonlinear matrix equation of the form (1.2).

In this paper, we study the perturbation analysis for the eigenvalues and eigenvectors of matrix polynomials of degree $m$. A backward error analysis for perturbed nonlinear eigenvalue problems has been developed in $[6,7,8,15]$ and the references therein. We discuss the homogeneous framework for the construction of the nearest perturbed palindromic nonlinear matrix equation such that an approximate eigenpair becomes an exact eigenpair of an appropriately perturbed palindromic problem with rational coefficients. This study extends the previous work on nonlinear eigenvalue problems with symmetric, skew-symmetric, Hermitian, and skew-Hermitian coefficients; see [6]. Due to the presence of the eigenvalues 0 and $\infty$ in palindromic nonlinear eigenvalue problems, we deal with the problem in a homogeneous framework of the form (1.2), which may contains both 0 and $\infty$ as eigenvalues. In contrast to the previous work [3, 2], here we consider the homogeneous form (1.2), where eigenvalues are represented as pairs $(c, s) \in \mathcal{R}$, and $c \neq 0$ corresponds to finite eigenvalues $\lambda=s / c$, while $(0,1)$ corresponds to the eigenvalue at $\infty$. This leads to the delay differential equation that generates a nonlinear matrix equation, which is further converted into palindromic/antipalindromic nonlinear eigenvalue problems depending on the coefficient matrices; see [16]. See $[12,13,14]$ for a nonlinear eigenvalue problem that occurs in the investigation of a delay differential equation that is converted into a quadratic matrix equation. However, due to rounding errors we avoid this transformation, so we find the results for the case of nonlinear eigenvalue problems and then treat the polynomial eigenvalue problem as a special case. The present results are compared with available results. The highlights of this work are as follows:

1. Formulae are developed for the nearest perturbations of nonlinear matrix equations of $T$-palindromic/T-anti-palindromic-type such that an approximate eigenpair becomes an exact eigenpair of appropriately perturbed $T$-palindromic/T-anti-palindromic nonlinear matrix equations. The above construction is also valid for the case of $T$-palindromic/T-anti-palindromic matrix polynomials.

2. Formulae are developed for the nearest perturbations of nonlinear matrix equations of type (1.2) having $H$-palindromic/ $H$-anti-palindromic structure such that an approximate eigenpair becomes an exact eigenpair of appropriately perturbed $H$-palindromic/H-anti-palindromic-structured nonlinear eigenvalue problems of type (1.2). The above construction is also valid for the case of $H$-palindromic $H$ anti-palindromic polynomials. If $(\lambda, \mu)=(-1,1),(1,-1)$ for the $H$-palindromic case and $(\lambda, \mu)=(1,1),(-1,-1)$ for the $H$-anti-palindromic case, then the structured backward error with respect to the 2 -norm is equal to $\sqrt{2}$ times the unstructured backward error for the case of the Frobenius norm of matrices. Also note that this is the same for the T-palindromic/T-anti-palindromic matrix polynomial case. 
The organization of the paper is as follows: Section 2 introduces notation and preliminaries. In Section 3, minimum structured perturbations of T-palindromic and T-anti-palindromic eigenvalue problems are derived, and the relations between the structured backward error and the unstructured backward error are discussed. In addition to that, minimum structured perturbation of $H$-palindromic and $H$-anti-palindromic eigenvalue problems for nonlinear matrix equations are derived, and the relationship between the structured backward error and the unstructured backward error are presented. In Section 4, formulae for the case of polynomial matrix equations and comparisons with known results in the literature are discussed. In Section 5, we discuss structured and unstructured pseudospectra for nonlinear matrix equations. Finally, Section 6 illustrates the pseudospectra associated with the above problems.

2. Preliminaries. We use the following notation: let $w \in \mathbb{R}^{m}$ be a nonnegative vector, and for $x \in \mathbb{C}^{m}$, we define the weighted norm $\|x\|_{w, 2}:=\left\|\left[w_{1} x_{1}, w_{2} x_{2}, \ldots, w_{m} x_{m}\right]^{T}\right\|_{2}$, where \|\|$_{2}$ denotes the Euclidean norm in $\mathbb{C}^{m}$. If $w$ is strictly positive, then this defines a norm/seminorm. For a nonnegative vector $w \in \mathbb{R}^{m}$, we define the inverse of $w$ via $w^{-1}:=\left[w_{1}^{-1}, w_{2}^{-1}, \ldots, w_{m}^{-1}\right]^{T}$, where $w_{i}^{-1}:=0$ if $w_{i}=0$. For $A \in \mathbb{C}^{m \times m}$, we define $A^{H}$ as the conjugate transpose of $A$ and $A^{T}$ as the transpose of $A$. $I$ is an identity matrix. For $x \in \mathbb{C}^{m}$ with $x^{H} x=1$, we define $P_{x}=I-x x^{H}$. The spectral and Frobenius norm on $\mathbb{C}^{m \times m}$ are defined by $\|A\|_{2}:=\max _{\|x\|=1}\|A x\|_{2}$ and $\|A\|_{F}:=\left(\operatorname{trace} A^{H} A\right)^{1 / 2}$, respectively.

Define the partial gradient $\nabla_{i}\|z\|_{w, 2}$ of a map $\mathbb{C}^{m} \backslash\{0\} \rightarrow \mathbb{R}, z \mapsto\|z\|_{w, 2}$ to be the gradient of the map $\mathbb{C} \backslash\{0\} \rightarrow \mathbb{R}, z_{i} \mapsto\left\|\left[z_{1}, \ldots, z_{m}\right]^{T}\right\|_{w, 2}$ with the variables $z_{j}(1 \leq j \leq n, j \neq i)$ fixed as constants. The gradient of the map $\mathbb{C}^{m} \backslash\{0\} \rightarrow \mathbb{R}, z \mapsto\|z\|_{w, 2}$, is then defined as

$$
\nabla\left(\|z\|_{w, 2}\right)=\left[\nabla_{1}\|z\|_{w, 2}, \nabla_{2}\|z\|_{w, 2}, \ldots, \nabla_{m}\|z\|_{w, 2}\right]^{T} \in \mathbb{C}^{m} .
$$

Let $M \otimes f(c, s)=\sum_{j=1}^{m} f_{j}(c, s) M_{j}, M_{j} \in \mathbb{C}^{n \times n}$. Define

$$
\operatorname{rev}\left(M^{T} \otimes f\right)=M \otimes f(s, c), \quad \text { and } \quad M^{\star} \otimes f=\sum_{j=1}^{m} f_{j} M_{j}^{\star}, \quad \star \in\{T, H\} .
$$

We use the symbol $\star$ as an abbreviation for the transpose $T$ in the real case and the transpose $T$ or conjugate transpose $*$ in the complex case. For $M=\left[M_{0}, M_{1}, \ldots, M_{n}\right]^{T}$, define the norm of $M$ with respect to the weight vector $w=\left[w_{0}, w_{1}, \ldots, w_{n}\right]^{T}, w_{i}>0$, as

$$
\|M\|_{w, 2}=\left\|\left(M_{0}, M_{1}, \ldots, M_{n}\right)\right\|_{w, 2}=\left(w_{0}^{2}\left\|M_{0}\right\|^{2}+\cdots+w_{n}\left\|M_{n}\right\|^{2}\right)^{1 / 2} .
$$

The following choices are considered in this paper:

1. The choice $f_{j}=\gamma^{j}$ encodes the non-homogeneous polynomial case $f_{j}(\gamma)=\gamma^{j}$.

2. The choice $f_{j}=\lambda^{j} \mu^{m-j}$ encodes the homogeneous polynomial case $f_{j}(\lambda, \mu)$.

3 . The choice $f_{j}=f_{j}(\lambda)$ encodes the non-homogeneous case with general functions $f_{j}$.

4. The choice $f_{j}=f_{j}(\lambda, \mu)$ encodes the homogeneous case with general functions $f_{j}$.

Under this assumption, a given eigenvalue $(\lambda, \mu)$ or $(\gamma)$ can be expressed by functions $f_{j}(\lambda, \mu)$ or $f_{j}(\gamma)$, respectively, in a uniform way. Thus, we have the following structured matrix equation of the form (1.2) that generalizes the case of matrix polynomial given in [16]:

\begin{tabular}{|l|l|}
\hline *-palindromic & $\operatorname{rev}(M \otimes f)=M^{*} \otimes f$ or $M_{j}=M_{m-j}^{*}$ \\
\hline *-anti-palindromic & $\operatorname{rev}(M \otimes f)=-M^{\star} \otimes f$ or $M_{j}=-M_{m-j}^{\star}$ \\
\hline
\end{tabular}


Moreover, we often use the product notation as described in [6] in the following way:

$$
(M \otimes f(c, s)) x=0, \quad(M \otimes f(\lambda)) x=0,
$$

respectively, with tuples of matrices $M:=\left(M_{0}, M_{1}, \ldots, M_{m}\right) \in\left(\mathbb{C}^{n \times n}\right)^{m+1}$ and functions $f(c, s):=\left(f_{0}(c, s), f_{1}(c, s), \ldots, f_{m}(c, s)\right) \in \mathbb{C}^{m+1}$ or $f(\lambda):=\left(f_{0}(\lambda), f_{1}(\lambda), \ldots, f_{m}(\lambda)\right)$ $\in \mathbb{C}^{m+1}$, respectively. The smallest perturbations $\Delta M$ with respect to the 2-norm and the Frobenius norm such that

$$
((M+\Delta M) \otimes f) x=\left(\sum_{j=0}^{m}\left(M_{j}+\Delta M_{j}\right) f_{j}\right) x=0 .
$$

leads to the backward errors, which are defined as follows:

DEFINITION 2.1. Let $M \in M_{m+1}^{\mathrm{S}}\left(\mathbb{C}^{n \times n}\right)$. Then the backward error of the matrix equation (1.1) is given by

$\eta_{w, 2}^{\mathbf{S}}(f, x, M):=\min \left\{\|\Delta M\|_{w, 2}: \Delta M \in M_{m+1}^{\mathbf{S}}\left(\mathbb{C}^{n \times n}\right): \operatorname{det}((M+\Delta M) \otimes f)=0\right\}$.

The backward error for the unstructured $M \in M_{m+1}\left(\mathbb{C}^{n \times n}\right)$ is given by

$$
\eta_{w, 2}(f, x, M):=\min \left\{\|\Delta M\|_{w, 2}: \Delta M \in M_{m+1}\left(\mathbb{C}^{n \times n}\right): \operatorname{det}((M+\Delta M) \otimes f)=0\right\} .
$$

For a given approximate eigenpair $((\lambda, \mu), x)$ or $(\gamma, x)$ of $M \otimes f$, a minimal perturbation $\Delta M \in\left(\mathbb{C}^{n \times n}\right)^{m+1}$ with respect to the Frobenius norm can be determined such that it becomes an exact eigenpair of $(M+\Delta M) \otimes f$. This follows from the following proposition.

PROPOSITION 2.2 ([6, Theorem 2.2]). Let $M \in M_{m+1}\left(\mathbb{C}^{n \times n}\right)$ be of the form (1.2). Let $f_{j}$ be sufficiently smooth functions. For a given approximate eigenvalue $(\lambda, \mu)$ or $(\gamma)$, set

$$
H_{w, 2}(f)=\left\|\left[w_{1} f_{1}, \ldots, w_{m} f_{m}\right]^{T}\right\|_{2} .
$$

Then the backward error, i.e., the size of the smallest perturbation that makes $(\lambda, \mu)$ or $(\gamma)$ an eigenvalue of the perturbed problem satisfies

$$
\eta_{w, 2}(f, x, M)=\min _{\|x\|=1} \frac{\|(M \otimes f) x\|}{H_{w^{-1}, 2}(f)}=\frac{\left\|(M \otimes f)^{-1}\right\|^{-1}}{H_{w^{-1}, 2}(f)} .
$$

DEFINITION 2.3. Let $M \in M_{m+1}\left(\mathbb{C}^{n \times n}\right)$. Consider a nonlinear matrix equation of the form (1.2). Then the structured and unstructured $\epsilon$-pseudospectrum with respect to the Frobenius norm are given by

$$
\begin{aligned}
& \Lambda_{\epsilon, w}^{\mathrm{S}}(M)=\left\{\psi \in \mathbb{C}^{n+1}: \eta_{w, 2}^{\mathbf{S}}(\psi, x, M) \leq \epsilon\right\}, \quad M \in M_{m+1}^{\mathbf{S}}\left(\mathbb{C}^{n \times n}\right), \quad \text { and } \\
& \Lambda_{\epsilon, w}(M)=\left\{\psi \in \mathbb{C}^{n+1}: \eta_{w, 2}(\psi, x, M) \leq \epsilon\right\}, \quad M \in M_{m+1}\left(\mathbb{C}^{n \times n}\right),
\end{aligned}
$$

respectively.

2.1. Some properties of partial gradients. In this section, some properties of the functions $H_{w, 2}$ and $G_{w, 2}$ are derived which are required for the subsequent development in our theory. First, we provide the following proposition from $[4,5,8]$ :

Proposition 2.4. Consider the following map $H_{w, 2}: \mathbb{C}^{n} \backslash\{0\} \rightarrow \mathbb{R}$ given by $H_{w, 2}(z):=\left\|\left[z_{1}, \ldots, z_{n}\right]^{T}\right\|_{w, 2}$. Then $H_{w, 2}$ is differentiable on $\mathbb{C}^{n}$, and the partial gradient satisfies $\nabla_{j} H_{w, 2}(z)=\frac{w_{j}^{2} z_{j}}{H_{w, 2}(z)}, 1 \leq j \leq n$. Furthermore,

$$
\sum_{j=1}^{m} z_{j} \frac{\bar{\nabla}_{j} H_{w, 2}(z)}{H_{w, 2}(z)}=1, \quad \sum_{j=1}^{m} w_{j}^{-2}\left|\nabla_{j} H_{w, 2}(z)\right|^{2}=1 .
$$


Consider the nonnegative vector $w=\left[w_{1}, w_{2}, \ldots, w_{m}\right] \in \mathbb{R}^{m}$, where $w_{m}=w_{m-j}$. Let $H_{w, 2}=\left\|\left[z_{0}, z_{1}, \ldots, z_{m}\right]^{T}\right\|_{w, 2}=\left(\sum_{j=0}^{m} w_{j}^{2}\left|z_{j}\right|^{2}\right)^{1 / 2}$, and let

$$
w_{j} y_{j}^{\epsilon}=\frac{w_{j} z_{j}+\epsilon w_{m-j} z_{m-j}}{2}, \epsilon= \pm 1
$$

Similar to Proposition 2.4, the following function $G_{w, 2}$, is needed for the perturbation theory of palindromic structures. Define

$$
\begin{aligned}
& \underset{\epsilon}{\mathbf{G}_{w, 2}: \mathbb{C}^{m+1} \backslash\{0\}} \rightarrow \mathbb{R} \\
& \mathbf{G}_{w, 2}\left(y^{\epsilon}\right)=\left\|\left(w_{0} y_{0}^{\epsilon}, w_{1} y_{1}^{\epsilon}, \ldots, w_{m} y_{m}^{\epsilon}\right)\right\|_{w, 2},
\end{aligned}
$$

where $y^{\epsilon}:=\left(y_{0}^{\epsilon}, y_{1}^{\epsilon}, \ldots, y_{m}^{\epsilon}\right) \in \mathbb{C}^{m+1}$ and the $y_{j}^{\epsilon}$ are defined in equation (2.1). Now we state the following result with the above setup.

PROPOSITION 2.5. Let

$$
H_{w, 2}(z)=\left\|\left[z_{1}, \ldots, z_{n}\right]^{T}\right\|_{w, 2}, \quad \text { and } \quad w_{j} y_{j}^{\epsilon}=\left(w_{j} z_{j}+\epsilon w_{m-j} z_{m-j}\right) / 2,
$$

where $w_{j}=w_{m-j}$, for $0 \leq j \leq m, \epsilon= \pm 1$. Consider

$$
\begin{aligned}
\mathbf{G}_{\epsilon, 2}: \mathbb{C}^{m+1} \backslash\{0\} & \rightarrow \mathbb{R} \\
y^{\epsilon} & \mapsto\left\|\left(w_{0} y_{0}^{\epsilon}, w_{1} y_{1}^{\epsilon}, \ldots, w_{m} y_{m}^{\epsilon}\right)\right\|_{w, 2} .
\end{aligned}
$$

Then $\mathbf{G}_{\epsilon, 2}$ is differentiable and $\nabla_{j} \mathbf{G}_{\epsilon, 2}\left(y^{\epsilon}\right)=\frac{w_{j}^{2} y_{j}^{\epsilon}}{\mathbf{G}_{\epsilon, 2}}$. Furthermore,

$$
\sum_{j=1}^{m} y_{j}^{\epsilon} \frac{\overline{\nabla_{j} \mathbf{G}_{\epsilon, 2}}\left(y^{\epsilon}\right)}{{\underset{\mathbf{G}}{\epsilon, 2}}_{w}\left(y^{\epsilon}\right)}=1 \quad \text { and } \quad \sum_{j=1}^{m} w_{j}^{-2}\left|\nabla_{j} \underset{\epsilon}{\mathbf{G}_{w, 2}}\left(y^{\epsilon}\right)\right|^{2}=1 .
$$

Proof. The proof follows from Proposition 2.4.

Proposition 2.6. Consider $y \in \mathbb{C}^{m+1} \backslash\{0\}$ and $w \in \mathbb{R}^{m+1}$. For $\epsilon= \pm 1$, let $\mathbf{G}_{\epsilon, 2}\left(y^{\epsilon}\right)=\left\|\left(w_{0} y_{0}^{\epsilon}, w_{1} y_{1}^{\epsilon}, \ldots, w_{m} y_{m}^{\epsilon}\right)\right\|_{w, 2}$. Then

$$
\sum_{j=0}^{m} f_{j} \frac{\nabla_{j} \mathbf{G}_{w, 2}}{\mathbf{G}_{\epsilon, 2}}=1
$$

where $H_{w, 2}=\left\|\left(w_{0} f_{0}, \ldots, w_{m} f_{m}\right)\right\|, f_{j}=f_{j}(\lambda, \mu)$ has no poles, and $y_{j}^{\epsilon}=\left(f_{j}+\epsilon f_{m-j}\right) / 2$, $f_{j} \in \mathbb{C}, j=0,1, \ldots, m$.

Proof. We have that $y_{j}^{\epsilon}=\left(f_{j}+\epsilon f_{m-j}\right) / 2,0 \leq j \leq m$.

Case I $(a)$ : let $m$ be even and $\epsilon=+1$. Then we have following identity:

$$
\begin{gathered}
\sum_{j=0}^{m} f_{j} \frac{\overline{\nabla_{j} \mathbf{G}_{\epsilon, 2}}}{\mathbf{G}_{\epsilon}}=\sum_{j=0}^{m} f_{j} \frac{w_{j}^{2} \overline{y_{j}^{\epsilon}}}{\left(\mathbf{G}_{\epsilon, 2}\right)^{2}}=w_{0}^{2} \frac{\overline{y_{0}^{\epsilon}}}{\left(\mathbf{G}_{\epsilon, 2}\right)^{2}}\left(f_{0}+f_{m}\right) / 2+\cdots+\frac{f_{\tilde{m}}}{2} w_{\tilde{m}}^{2} \frac{\overline{y_{\tilde{m}}^{\epsilon}}}{\left(\mathbf{G}_{\epsilon, 2}\right)^{2}} \\
+\cdots+w_{m}^{2} \frac{\overline{y_{m}^{\epsilon}}}{\left(\mathbf{G}_{\epsilon, 2}\right)^{2}}\left(f_{m}+f_{0}\right) / 2=\sum_{j=0}^{m} \frac{w_{j}^{2}\left|y_{j}^{\epsilon}\right|^{2}}{\left(\mathbf{G}_{\epsilon, 2}\right)^{2}}=1 .
\end{gathered}
$$


Case I(b): Let $m$ be even and $\epsilon=-1$. Then we can write

$$
\begin{aligned}
& \sum_{j=0}^{m} f_{j} \frac{\overline{\nabla_{j} \mathbf{G}_{\epsilon, 2}}}{\mathbf{G}_{\epsilon}}=\sum_{j=0}^{m} f_{j} \frac{w_{j}^{2} \overline{y_{j}^{\epsilon}}}{\left(\mathbf{G}_{\boldsymbol{\epsilon}}\right.}=w_{0}^{2} \frac{\overline{y_{0}^{\epsilon}}}{\left(\mathbf{G}_{w, 2}\right)^{2}}\left(f_{0}-f_{m}\right) / 2 \\
& +\cdots+w_{m-j}^{2} \frac{\overline{y_{m-j}^{\epsilon}}}{\left(\mathbf{G}_{w, 2}\right)^{2}}\left(f_{j}-f_{m-j}\right) / 2 \\
& +\cdots+w_{m}^{2} \frac{\overline{y_{m}^{\epsilon}}}{\left(\mathbf{G}_{\epsilon, 2}\right)^{2}}\left(f_{m}-f_{0}\right) / 2=\sum_{j=0}^{m} \frac{w_{j}^{2}\left|y_{j}^{\epsilon}\right|^{2}}{\left(\mathbf{G}_{w, 2}\right)^{2}}=1 .
\end{aligned}
$$

Case II: For $m$ odd with $y_{j}^{\epsilon}=\left(f_{j}+\epsilon f_{m-j}\right) / 2, f_{j} \in \mathbb{C}$, we have the following expressions: Case II $(a)$ : Let $m$ be odd and $\epsilon=+1$ :

$$
\sum_{j=0}^{m} f_{j} \frac{\overline{\nabla_{j} \mathbf{G}_{w, 2}}}{\mathbf{G}_{\epsilon, 2}}=\sum_{j=0}^{m} f_{j} \frac{w_{j}^{2} \overline{y_{j}^{\epsilon}}}{\left(\mathbf{G}_{\epsilon, 2}\right)^{2}}=\sum_{j=0}^{m} \frac{w_{j}^{2}\left|y_{j}^{\epsilon}\right|^{2}}{\left(\mathbf{G}_{w, 2}\right)^{2}}=1 .
$$

Case II $(b)$ : Let $m$ be odd and $\epsilon=-1$ :

$$
\sum_{j=0}^{m} f_{j} \frac{\overline{\nabla_{j} \mathbf{G}_{w, 2}}}{\mathbf{G}_{\epsilon, 2}}=\sum_{j=0}^{m} f_{j} \frac{w_{j}^{2} \overline{y_{j}^{\epsilon}}}{\left(\mathbf{G}_{\epsilon, 2}\right)^{2}}=\sum_{j=0}^{m} \frac{w_{j}^{2}\left|y_{j}^{\epsilon}\right|^{2}}{\left(\mathbf{G}_{w, 2}\right)^{2}}=1 .
$$

3. Backward errors for the palindromic rational eigenvalue problem. In this section we discuss the perturbation analysis of $T$-palindromic/ $T$-anti-palindromic and $\mathrm{H}$-palindromic/ $\mathrm{H}$-anti-palindromic problems. In our construction, we find a lots of similarities in the perturbation analysis when we move from palindromic problems to anti-palindromic structures. We use the function $\mathbf{G}_{\epsilon, 2}\left(y^{\epsilon}\right)$ as defined in equation (2.2), where $y^{\epsilon}:=\left(y_{0}^{\epsilon}, y_{1}^{\epsilon}, \ldots, y_{m}^{\epsilon}\right) \in \mathbb{C}^{m+1}$. For $\epsilon=1$, we derive the perturbation for the symmetric case, and when $\epsilon=-1$ we derive the perturbation for the anti-palindromic case. Throughout the paper we use $Z_{M_{j}}^{\epsilon}$ defined as follows:

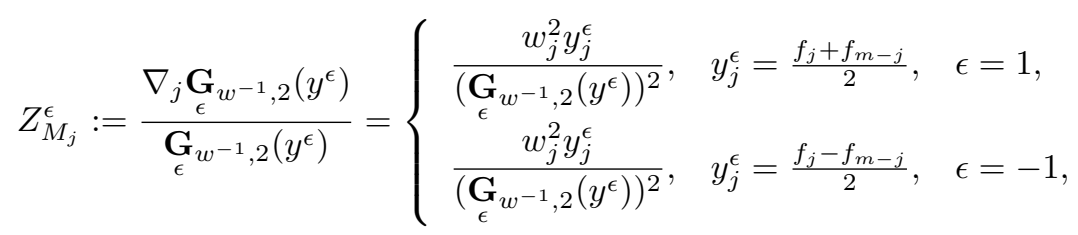

where $\left.\mathbf{G}_{\epsilon, 2}\left(y^{\epsilon}\right)\right)=\left\|\left(w_{0} y_{0}^{\epsilon}, w_{1} y_{1}^{\epsilon}, \ldots, w_{m} y_{m}\right)\right\|_{w, 2}, y^{\epsilon}=\left(y_{0}^{\epsilon}, y_{1}^{\epsilon}, \ldots, y_{m}^{\epsilon}\right) \in \mathbb{C}^{m+1}$, and $f=\left(f_{0}, f_{1}, \ldots, f_{m}\right) \in \mathbb{C}^{m+1}$.

With this result, we now derive the perturbation formula for the case of $T$-palindromic and T-anti-palindromic problems. Our derivation gives a perturbation of the T-palindromic problem when $\epsilon=1$ and a perturbation of T-anti-palindromic problem for $\epsilon=-1$. For a perturbation for homogeneous structures, we use the notation $((\lambda, \mu), x)$, and similarly, for non-homogeneous structures we use $(\gamma, x)$. Here $((\lambda, \mu), x)$ is an approximate eigenpair of equation (1.2), and $(\gamma, x)$ is an approximate eigenpair of equation (1.2) for $s=1$.

THEOREM 3.1. Let $M \in M\left(\mathbb{C}^{n \times n}\right)$ be a T-palindromic/T-anti-palindromic rational eigenvalue problem of the form (1.2). Let $((\lambda, \mu), x)$ or $(\gamma, x)$ be an approximate eigenpair of (1.2). Construct $\tau=\left(\tau_{0}, \ldots, \tau_{m}\right) \in \mathbb{C}^{m+1}, \tau_{j}=f_{j}(\lambda, \mu)$ or $\tau_{j}=f_{j}(\gamma)$. Set 
$k=-(M \otimes \tau) x, Z_{M_{j}}^{\epsilon}$ as in (3.1), and $P_{x}=I-x x^{H}\left(x \in \mathbb{C}^{n}\right)$. Then we have

$$
\eta_{w, F}^{\mathbf{S}}(\tau, x, M)=\left\{\sqrt{\frac{2\|k\|_{2}^{2}-\left|x^{T} k\right|^{2}}{\mathbf{G}_{\epsilon}^{2} w^{-1}, 2}}, \quad(\lambda, \mu) \text { is not a pole of } f_{j} .\right.
$$

Introduce the perturbation matrices, for $j=1,2, \ldots, m$,

$$
\Delta M_{j}:=\bar{Z}_{M_{j}}^{\epsilon} \bar{x} x^{T} k x^{H}+\epsilon \bar{Z}_{M_{m-j}}^{\epsilon} P_{x}^{T} k x^{T}+\bar{Z}_{M_{j}}^{\epsilon} \bar{x} k^{T} P_{x}, \quad \forall j .
$$

Then there exists $M \otimes f=\sum_{j=1}^{m} f_{j} \Delta M_{j}$ such that $(M+\Delta M) \otimes \tau=0$.

Proof. Consider

$$
\widetilde{\Delta M_{j}}=U^{T} \Delta M_{j} U=\left[\begin{array}{cc}
a_{j, j} & a_{j}^{T} \\
b_{j} & X_{j}
\end{array}\right], \quad b_{j}=\epsilon a_{m-j}, a_{j j}=\epsilon a_{m-j, m-j}, X_{m-j}=\epsilon X_{j}^{T},
$$

such that $\widetilde{\Delta M_{m-j}}=\epsilon{\widetilde{\Delta M_{j}}}^{T}(0 \leq j \leq m)$, where $U=\left[\begin{array}{ll}x & U_{1}\end{array}\right]$ is a unitary matrix. Since $((M+\Delta M) \otimes \tau) x=0$, it follows that $(M \otimes \tau+\Delta M \otimes \tau) x=0$, and hence $k=(\Delta M \otimes \tau) x$. Now we construct a unitary matrix $U$ which has $x$ as its first column, i.e., $U=\left[\begin{array}{ll}x & U_{1}\end{array}\right] \in \mathbb{C}^{n \times n}$. Then $\bar{U}(\widetilde{\Delta M} \otimes \tau) U^{H}=\bar{U} U^{T}(\Delta M \otimes \tau) U^{H} U=\Delta M \otimes \tau$, and hence $\bar{U}(\widetilde{\Delta M} \otimes \tau) U^{H} x=(\Delta M \otimes \tau) x=k$, which implies

$$
(\widetilde{\Delta M} \otimes \tau) U^{H} x=U^{T} k=\left[\begin{array}{c}
x^{T} k \\
U_{1}^{T} k
\end{array}\right]
$$

Then we have

$$
\left[\begin{array}{c}
a_{00} \tau_{0}+a_{11} \tau_{1}+\cdots+a_{m m} \tau_{m} \\
b_{0} \tau_{0}+b_{0} \tau_{1}+\cdots+b_{m} \tau_{m}
\end{array}\right]=\left[\begin{array}{c}
x^{T} k \\
U_{1}^{T} k
\end{array}\right]
$$

From the previous equation we may conclude the following equality:

$$
\left[\begin{array}{c}
\sum_{j=0}^{m} \frac{\left(\tau_{j}+\epsilon \tau_{m-j}\right)}{2} a_{j j} \\
\sum_{j=0}^{m} \frac{\left(\tau_{j}+\epsilon \tau_{m-j}\right)}{2} b_{j}
\end{array}\right]=\left[\begin{array}{c}
x^{T} k \\
U_{1}^{T} k
\end{array}\right] .
$$

Then we have

$$
\sum_{j=0}^{m} \frac{\tau_{j}}{2 w_{j}} w_{j} b_{j}+\epsilon \sum_{j=0}^{m} \frac{\tau_{m-j}}{2 w_{m-j}} w_{m-j} b_{m-j}=U_{1}^{T} k
$$

and

$$
\begin{aligned}
& \sum_{j=0}^{m} \frac{\tau_{j}}{2 w_{j}} w_{j} a_{j, j}+\epsilon \sum_{j=0}^{m} \frac{\tau_{m-j}}{2 w_{m-j}} w_{m-j} a_{m-j, m-j}=x^{H} k, \\
& \sum_{j=0}^{m} \frac{\beta_{j}\left(\tau_{j}+\epsilon \tau_{m-j}\right)}{2 \beta_{j}} a_{j j}=x^{T} k, \beta_{j}=w_{j} w_{m-j} .
\end{aligned}
$$


Using the least-squares method, the minimum-norm solutions are given by

$$
b_{j}=\frac{\overline{\nabla_{j} \mathbf{G}_{w^{-1}, 2}}}{\mathbf{G}_{w^{-1}, 2}} U_{1}^{T} k, \quad \text { and } \quad a_{j j}=\frac{\overline{\nabla_{j} \mathbf{G}_{w^{-1}, 2}}}{\mathbf{G}_{\epsilon}} x^{T} k .
$$

Since we have $b_{j}=\epsilon a_{m-j}$, it follows that

$$
\Delta M_{j}=\bar{U}\left[\begin{array}{cc}
\bar{Z}_{M_{j}}^{\epsilon} x^{T} k & \epsilon \bar{Z}_{M_{m-j}}\left(U_{1}^{T} k\right)^{T} \\
\bar{Z}_{M_{j}} U_{1}^{T} k & D_{j, j}
\end{array}\right] U^{H}
$$

where

$$
\begin{aligned}
\Delta M_{m-j} & =\epsilon \Delta M_{j}^{T}, \quad \text { and } \\
\Delta M_{j} & =\bar{Z}_{M_{j}}^{\epsilon} \bar{x} x^{T} k x^{H}+\epsilon \bar{Z}_{M_{m-j}} \bar{x} k^{T} P_{x}+\bar{Z}_{M_{j}} P_{x}^{T} k x^{H}+\overline{U_{1}} D_{j j} U_{1}^{T} .
\end{aligned}
$$

Choosing $D_{j j}=0$ we obtain

$$
\Delta M_{j}=\bar{Z}_{M_{j}}^{\epsilon} \bar{x} x^{T} k x^{H}+\epsilon \bar{Z}_{M_{m-j}} \bar{x} k^{T} P_{x}+\bar{Z}_{M_{j}} P_{x}^{T} k x^{H} .
$$

Now,

$$
\begin{aligned}
((M+\Delta M) \otimes \tau) x & =-k+\sum_{j=0}^{m} \tau_{j}\left[\bar{Z}_{M_{j}}^{\epsilon} \bar{x} x^{T} k x^{H}+\epsilon \bar{Z}_{M_{j}}^{\epsilon} \bar{x} k^{T} P_{x}+\bar{Z}_{M_{j}}^{\epsilon} P_{x}^{T} k x^{H}\right] x \\
& =-k+\sum_{j=0}^{m} \tau_{j}\left[\bar{Z}_{M_{j}}^{\epsilon} \bar{x} x^{T} k+\bar{Z}_{M_{j}}^{\epsilon} P_{x}^{H} k\right] \\
& =-k+\bar{x} x^{T} k+\left(I-\bar{x} x^{T}\right) k=0 .
\end{aligned}
$$

Thus, the backward error is given by

$$
\eta_{w, F}^{\mathbf{S}}(\tau, x, M)=\|\Delta M\|_{w, F}^{2}=\sqrt{\sum_{j=0}^{m}\left\|\Delta M_{j}\right\|^{2}}=\sqrt{\frac{2\|k\|_{2}^{2}-\left|x^{T} k\right|^{2}}{\mathbf{G}_{\epsilon}^{2} w^{-1}, 2}} .
$$

Similar to the previous theorem we derive formulae for the $H$-palindromic and $H$-antipalindromic cases. With the approximate eigenpairs we construct $\tau \in \mathbb{C}^{m+1}$.

THEOREM 3.2. Let $M \in M_{m+1}\left(\mathbb{C}^{n \times n}\right)$ be a H-palindromic/H-anti-palindromic structure of the form (1.2). Let $((\lambda, \mu), x)$ or $(\gamma, x)$ be an approximate eigenpair of (1.2). Construct $\tau=\left(\tau_{0}, \ldots, \tau_{m}\right) \in \mathbb{C}^{m+1}$, where $\tau_{j}=f_{j}(\lambda, \mu)$ or $\tau_{j}=f_{j}(\gamma)$. Let $k=-(M \otimes \tau) x, Z_{M_{j}}^{\epsilon}$ as in (3.1), and $P_{x}=I-x x^{H}$. Introduce the perturbation matrices

$$
\Delta M_{j}=\bar{Z}_{M_{j}}^{\epsilon} \bar{x} x^{H} k x^{H}+\epsilon \bar{Z}_{M_{m-j}}^{\epsilon} P_{x}^{H} k x^{H}+\bar{Z}_{M_{j}}^{\epsilon} \bar{x} k^{H} P_{x},
$$

and if $\tau_{j} \in \mathbb{C}$, introduce

$$
\Delta M_{j}=\beta_{j}^{-1} t_{j}^{\epsilon} x x^{H}+\epsilon \bar{Z}_{M_{m-j}}^{\epsilon} x k^{H} P_{x}+Z_{M_{j}}^{\epsilon} P_{x}^{H} k x^{H},
$$

where $\beta_{j}=w_{j}^{-1} w_{m-j}^{-1}$.

Then there exists $\Delta M \in M_{m}\left(\mathbb{C}^{n \times n}\right)$ such that $((M+\Delta M) \otimes \tau) x=0$. The backward error is given by 


$$
\eta_{w, F}^{\mathbf{S}}(\tau, x, M)= \begin{cases}\sqrt{\frac{2\|k\|_{2}^{2}-\left|x^{H} k\right|^{2}}{\mathbf{G}_{\epsilon}^{2} w^{-1}, 2}} & \text { for } \tau_{j} \in \mathbb{R}, \\ \sqrt{\sum_{j=0}^{m}\left|\frac{t_{j}^{\epsilon}}{\beta_{j}}\right|^{2}+\frac{2\left(\|k\|_{2}^{2}-\left|x^{H} k\right|^{2}\right)}{\mathbf{G}^{2} w^{-1}, 2}} & \text { for } \tau_{j} \in \mathbb{C} .\end{cases}
$$

In this theorem, $t_{j}$ is a component of a vector $t$, where

$$
t=T^{+}\left[\begin{array}{l}
\Re\left(x^{H} k\right) \\
\Im\left(x^{H} k\right)
\end{array}\right], \quad \text { with } T:=\left[\begin{array}{ccc}
\frac{\Re\left(y_{0}^{\epsilon}\right)}{2 \beta_{0}} & \ldots & \frac{\Re\left(y_{m}^{\epsilon}\right)}{2 \beta_{m}} \\
\frac{\Im\left(y_{0}^{\epsilon}\right)}{2 \beta_{0}} & \ldots & \frac{\Im\left(y_{m}^{\epsilon}\right)}{2 \beta_{m}}
\end{array}\right], y_{j}^{\epsilon}=\frac{\tau_{j}+\epsilon \tau_{m-j}}{2}, \epsilon= \pm 1 .
$$

Proof. Case I: Let $\tau_{j} \in \mathbb{R}$, then the proof follows from the previous Theorem 3.1.

Case II: If $\tau_{j} \in \mathbb{C}$. Then from (3.2) we have

$$
\sum_{j=0}^{m} \frac{\tau_{j}}{2 w_{j}} w_{j} a_{j, j}+\sum_{j=0}^{m} \frac{\tau_{m-j}}{2 w_{m-j}} w_{m-j} a_{m-j, m-j}=x^{H} k .
$$

Hence, the identity

$$
\left[\begin{array}{c}
\sum_{j=0}^{m} \frac{w_{m-j} w_{j} \Re\left(z_{j}\right)+\epsilon w_{j} w_{m-j} \Re\left(\tau_{m-j}\right)}{2\left(w_{j} w_{m-j}\right)} a_{j j} \\
\sum_{j=0}^{m} \frac{w_{j} \Im\left(\tau_{j}\right)+\epsilon w_{m-j} \Im\left(\tau_{m-j}\right)}{2\left(w_{j} w_{m-j}\right)} a_{j j}
\end{array}\right]=\left[\begin{array}{c}
\Re\left(x^{H} k\right) \\
\Im\left(x^{H} k\right)
\end{array}\right]
$$

shows that

$$
\left[\begin{array}{l}
\sum_{j=0}^{m} \beta_{j} \frac{\Re\left(\tau_{j}+\epsilon \tau_{m-j}\right)}{2 \beta_{j}} a_{j j} \\
\sum_{j=0}^{m} \beta_{j} \frac{\Im\left(\tau_{j}+\epsilon \tau_{m-j}\right)}{2 \beta_{j}} a_{j j}
\end{array}\right]=\left[\begin{array}{l}
\Re\left(x^{H} k\right) \\
\Im\left(x^{H} k\right)
\end{array}\right] \text { and }\left[\begin{array}{l}
\sum_{j=0}^{m} \frac{\beta_{j} \Re\left(y_{j}^{\epsilon}\right)}{\beta_{j}} a_{j j} \\
\sum_{j=0}^{m} \beta_{j} \frac{\Im\left(y_{j}^{\epsilon}\right)}{\beta_{j}} a_{j j}
\end{array}\right]=\left[\begin{array}{l}
\Re\left(x^{H} k\right) \\
\Im\left(x^{H} k\right)
\end{array}\right]
$$

where $y_{j}^{\epsilon}=\left(\tau_{j}+\epsilon \tau_{m-j}\right) / 2$. This implies

$$
\begin{aligned}
& {\left[\begin{array}{ccc}
\frac{\Re\left(y_{0}^{\epsilon}\right)}{2 \beta_{0}} & \ldots & \frac{\Re\left(y_{m}^{\epsilon}\right)}{2 \beta_{m}}
\end{array}\right]\left[\begin{array}{c}
\beta_{0} a_{00} \\
\vdots \\
\alpha_{m} a_{m m}
\end{array}\right]=\Re\left(x^{H} k\right),} \\
& {\left[\begin{array}{ccc}
\Im\left(y_{0}^{\epsilon}\right) \\
2 \beta_{0} & \ldots & \frac{\Im\left(y_{m}^{\epsilon}\right)}{2 \beta_{m}}
\end{array}\right]\left[\begin{array}{c}
\beta_{0} a_{00} \\
\vdots \\
\beta_{m} a_{m m}
\end{array}\right]=\Im\left(x^{H} k\right) .}
\end{aligned}
$$

Combining equation (3.3) and equation (3.4), we get

$$
\left[\begin{array}{ccc}
\frac{\Re\left(y_{0}^{\epsilon}\right)}{2 \beta_{0}} & \ldots & \frac{\Re\left(y_{m}^{\epsilon}\right)}{2 \beta_{m}} \\
\frac{\Im\left(y_{0}^{\epsilon}\right)}{2 \beta_{0}} & \ldots & \frac{\Im\left(y_{m}^{\epsilon}\right)}{2 \beta_{m}}
\end{array}\right]\left[\begin{array}{c}
\beta_{0} a_{00} \\
\vdots \\
\beta_{m} a_{m m}
\end{array}\right]=\left[\begin{array}{c}
\Re\left(x^{H} k\right) \\
\Im\left(x^{H} k\right)
\end{array}\right] .
$$


Solving the above equation with the least-squares method, we obtain the following:

$$
\left[\begin{array}{c}
\beta_{0} d_{00} \\
\vdots \\
\beta_{m} d_{m m}
\end{array}\right]=T^{+}\left[\begin{array}{c}
\Re\left(x^{H} k\right) \\
\Im\left(x^{H} k\right)
\end{array}\right]=t, \quad \text { where } \quad T:=\left[\begin{array}{ccc}
\frac{\Re\left(y_{0}^{\epsilon}\right)}{2 \beta_{0}} & \ldots & \frac{\Re\left(y_{m}^{\epsilon}\right)}{2 \beta_{m}} \\
\frac{\Im\left(y_{0}^{\epsilon}\right)}{2 \beta_{0}} & \ldots & \frac{\Im\left(y_{m}^{\epsilon}\right)}{2 \beta_{m}}
\end{array}\right] .
$$

Thus, we have

$$
\Delta M_{j}=\bar{U}\left[\begin{array}{cc}
\beta_{j} t_{j}^{\epsilon} & \epsilon \bar{Z}_{M_{m-j}^{\epsilon}\left(U_{1}^{H} k\right)^{H}} \\
\bar{Z}_{M_{j}}^{\epsilon} U_{1}^{H} k & D_{j, j}
\end{array}\right] U^{H} .
$$

Since $a_{j j}=a_{m-j, m-j}$, it follows that $\Delta M_{m-j}=\epsilon \Delta M_{j}^{H}$, where $\beta_{j}=w_{j} w_{m-j}$ and $\beta_{j}^{-1}:=w_{j}^{-1} w_{m-j}^{-1}$, for $j=0,1, \ldots, m$. Hence,

$$
\Delta M_{j}=\beta_{j}^{-1} t_{j}^{\epsilon} x x^{H}+\epsilon \bar{Z}_{M_{m-j}}^{\epsilon} x k^{H} P_{x}+\bar{Z}_{M_{j}}^{\epsilon} P_{x}^{H} k x^{H}+\overline{U_{1}} D_{j j} U_{1}^{H} .
$$

Choosing $D_{j j}=0$ yields

$$
\Delta M_{j}=\beta_{j}^{-1} t_{j}^{\epsilon} x x^{H}+\epsilon \bar{Z}_{M_{m-j}^{\epsilon}} x k^{H} P_{x}+\bar{Z}_{M_{j}}^{\epsilon} P_{x}^{H} k x^{H} .
$$

Now we can show that the above perturbation $\Delta M_{j}$ satisfy the following equation:

$$
\begin{aligned}
& ((M+\Delta M) \otimes \tau) x \\
& =-k+\sum_{j=0}^{m} \lambda^{m-j} \mu^{j}\left[\beta_{j}^{-1} t_{j} x x^{H}+\epsilon \bar{Z}_{M_{m-j}}^{\epsilon} x k^{H} P_{x}+\bar{Z}_{M_{j}}^{\epsilon} P_{x}^{H} k x^{H}\right] x \\
& =-k+\sum_{j=0}^{m} \tau_{j}\left[\beta_{j}^{-1} t_{j} x+\bar{Z}_{M_{j}}^{\epsilon} P_{x}^{H} k\right]=-k+\sum_{j=0}^{m} \tau_{j} \beta_{j}^{-1} t_{j} x+\left(I-x x^{H}\right) k \\
& =\sum_{j=0}^{m} \tau_{j} \beta_{j}^{-1} t_{j} x+\sum_{j=0}^{\tilde{m}} \tau_{j} x\left(x^{H} \Delta M_{j} x\right)=0 .
\end{aligned}
$$

Now this $\Delta M_{j}$ yields the perturbation for the backward error which is given by

$$
\eta_{w, 2}^{\mathbf{S}}(\tau, x, M)=\sqrt{\sum_{j=0}^{m}\left|\frac{t_{j}^{\epsilon}}{\beta_{j}}\right|^{2}+\frac{2\left(\|k\|_{2}^{2}-\left|x^{H} k\right|^{2}\right)}{\mathbf{G}_{\epsilon}^{2} w^{-1}, 2}} .
$$

REMARK 3.3. Note that $t_{j}^{\epsilon}=\epsilon t_{m-j}$ for an $H$-anti-palindromic structure. Thus, we have $\Delta M_{j}=\epsilon \Delta M_{m-j}^{H}$. Also it should be noted that when $\beta_{j}=0$, then $\beta_{j}^{-1}=0$, and this implies $w_{j}=w_{m-j}=0$. Hence there is no perturbation in the coefficient matrices $A_{j}$ and $A_{m-j}$ when $\beta_{j}=0$. For $*$-palindromic and $*$-anti-palindromic matrix equations of the form (1.1), this gives rise to a relation between the structured backward error and the unstructured backward error under certain condition which are stated in the next corollaries.

COROLlaRY 3.4. Let $M \in M_{m+1}\left(\mathbb{C}^{n \times n}\right)$ be $*$-palindromic of the form (1.1) with $G_{w^{-1,2}} \neq 0, H_{w^{-1,2}} \neq 0$ at $(\lambda, \mu) \in \mathbb{C}^{2} \backslash\{0\}$ or at $\gamma \in \mathbb{C}$.

- If $\Re\left(\tau_{j} \tau_{m-j}\right) \geq 0$, then $\mathbf{G}_{\epsilon} w^{-1,2} \geq \frac{1}{\sqrt{2}} H_{w^{-1}, 2}$ and $\eta_{w, F}^{\mathbf{S}}(\tau, x, M) \leq 2 \eta_{w, 2}(\tau, x, M)$.

- If $\Re\left(\tau_{j} \tau_{m-j}\right) \leq 0$, then $\mathbf{G}_{w^{-1}, 2} \leq \frac{1}{\sqrt{2}} H_{w^{-1}, 2}$ and $\eta_{w, F}^{\mathbf{S}}(\tau, x, M) \geq 2 \eta_{w, 2}(\tau, x, M)$.

COROLlary 3.5. Let $M \in M_{m+1}\left(\mathbb{C}^{n \times n}\right)$ be $*$-anti-palindromic of the form (1.1) with $G_{w^{-1}, 2} \neq 0, H_{w^{-1}, 2} \neq 0$ at $(\lambda, \mu) \in \mathbb{C}^{2} \backslash\{0\}$ or at $\gamma \in \mathbb{C}$.

- If $\Re\left(\tau_{j} \tau_{m-j}\right) \leq 0$, then $\mathbf{G}_{\epsilon} w^{-1,2} \geq \frac{1}{\sqrt{2}} H_{w^{-1}, 2}$ and $\eta_{w, F}^{\mathbf{S}}(\tau, x, M) \leq 2 \eta_{w, 2}(\tau, x, M)$.

- If $\Re\left(\tau_{j} \tau_{m-j}\right) \geq 0$, then $\mathbf{G}_{w^{-1}, 2} \leq \frac{1}{\sqrt{2}} H_{w^{-1}, 2}$ and $\eta_{w, F}^{\mathbf{S}}(\tau, x, M) \geq 2 \eta_{w, 2}(\tau, x, M)$. 
TABLE 4.1

Eigenvalues and eigenvectors of palindromic matrix polynomial of the form (4.1).

\begin{tabular}{c|c|c}
$\mathbf{S}$ & Eigenvalues & Eigenpair \\
\hline T-palindromic & $((\lambda, \mu),(\mu, \lambda))$ & $((\lambda, \mu), y, x),((\mu, \lambda), \bar{x}, \bar{y})$ \\
\hline H-palindromic & $((\lambda, \mu),(\bar{\mu}, \bar{\lambda}))$ & $((\lambda, \mu), y, x),((\bar{\mu}, \bar{\lambda}), x, y)$ \\
\hline T-anti-palindromic & $((\lambda, \mu),(\mu, \lambda))$ & $((\lambda, \mu), y, x),((\mu, \lambda), \bar{x}, \bar{y})$ \\
\hline H-anti-palindromic & $((\lambda, \mu),(\bar{\mu}, \bar{\lambda}))$ & $((\lambda, \mu), y, x),((\bar{\mu}, \bar{\lambda}), x, y)$
\end{tabular}

4. Backward errors for palindromic polynomial eigenvalue problems. Now we derive the formulae for the case of homogeneous matrix polynomials. For this we consider $f_{j}=c^{m-j} s^{j}, j=0,1, \ldots, m$, in equation (1.2) and obtain

$$
M \otimes f(c, s)=\sum_{j=0}^{m} c^{m-j} s^{j} M_{j}=M(c, s) .
$$

Now we discuss the simpler case. Consider the matrix polynomial of the form (4.1). A special case of Theorem 3.1 can be derived for homogeneous matrix polynomials. We get the following results.

THEOREM 4.1. Let $M \in M_{m+1}\left(\mathbb{C}^{n \times n}\right)$ be a T-palindromic/T-anti-palindromic matrix polynomials of the form (4.1). Let $k=-M(\lambda, \mu) x, Z_{M_{j}}^{\epsilon}$ as in (3.1), and $P_{x}=I-x x^{H}$. Then we have

$$
\eta_{w, F}^{\mathbf{S}}(\lambda, \mu, x, M)=\left\{\begin{array}{l}
\sqrt{\frac{2\|k\|_{2}^{2}-\left|x^{T} k\right|^{2}}{\mathbf{G}_{\epsilon}^{2} w^{-1}, 2}} \text { if }\left\{\begin{array}{l}
\lambda^{m} \mu^{m} \neq-1, \forall \text { odd } m, *=T, \epsilon=+1, \\
\lambda^{m} \mu^{m} \neq 1, \forall \text { odd } m, *=-T, \epsilon=-1, \\
\forall(\lambda, \mu) \text { with even } m, *=\epsilon T, \epsilon= \pm 1,
\end{array}\right. \\
\sqrt{2} \eta_{w, 2}(\lambda, \mu, x, M) \quad \text { if }\left\{\begin{array}{l}
\lambda^{m} \mu^{m}=-1, \text { m-odd }, *=T, \epsilon=+1, \\
\lambda^{m} \mu^{m}=1, \text { modd }, *=-T, \epsilon=-1,
\end{array}\right. \\
\leq 2 \eta_{w, 2}(\lambda, \mu, x, M) \text { if } \quad \begin{array}{l}
\Re\left(\lambda^{m} \mu^{m}\right)=0, *=\epsilon T, \epsilon= \pm 1, \forall m .
\end{array}
\end{array}\right.
$$

Introduce the perturbation matrices

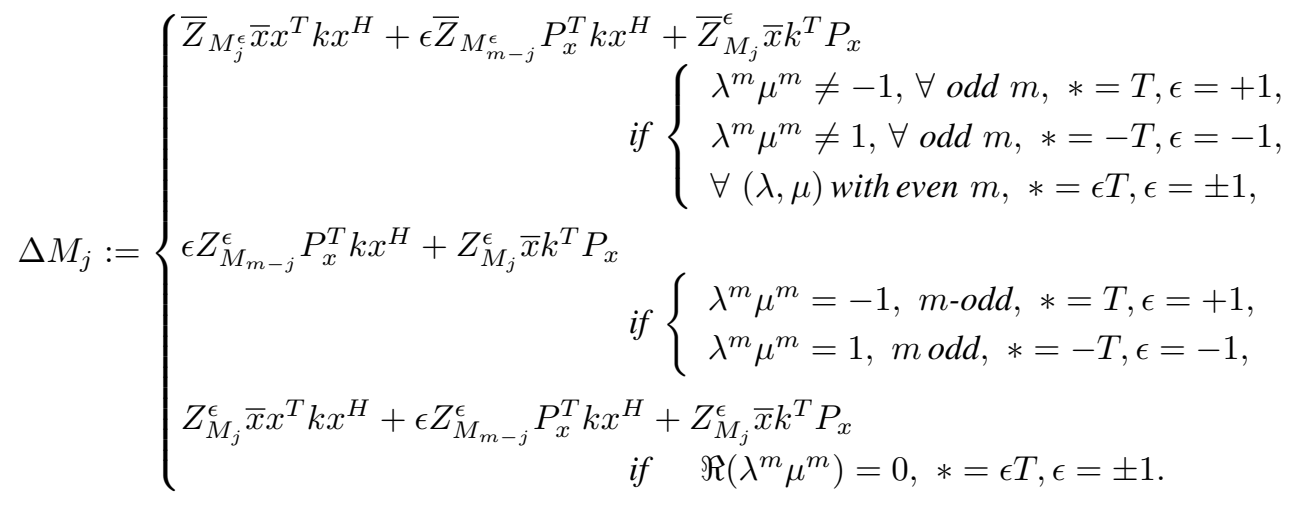

Then there exists $\Delta M$ such that $(M(\lambda, \mu)+\Delta M(\lambda, \mu)) x=0$.

Proof. The first part of the proof follows from the previous theorem. When $m$ is odd, we have the following two cases: 
Case A: $(\lambda, \mu)=(1,-1),(-1,1)$ for a $T$-palindromic structure.

Case B: $(\lambda, \mu)=(1,1),(-1,-1)$ for a $T$-anti-palindromic structure.

Hence the minimum-norm solution of $\sum_{j=0}^{m} f_{j} a_{j j}=x^{T} k$ is $a_{j j}=0$. Similarly for $\sum_{j=0}^{m} f_{j} b_{j}=U_{1}^{T} k$. Then we have for $j=0,1, \ldots, m$,

$$
\Delta M_{j}=\bar{U}\left[\begin{array}{cc}
0 & \epsilon Z_{M_{m-j}}^{\epsilon}\left(U_{1}^{T} k\right)^{H} \\
Z_{M_{j}}^{\epsilon} U_{1}^{T} k & D_{j, j}
\end{array}\right] U^{H}
$$

and $\Delta M_{j}=\epsilon Z_{M_{m-j}}^{\epsilon} \bar{x} k^{T} P_{x}+Z_{M_{j}}^{\epsilon} P_{x}^{T} k x^{H}$, and the backward error is given by

$$
\eta_{w, F}^{\mathbf{S}}(\lambda, \mu, x, M)=\sqrt{2} \frac{\|k\|}{H_{w^{-1}, 2}}=\sqrt{2} \eta_{w, 2}(\lambda, \mu, x, M) .
$$

COROLlary 4.2. For a T-palindromic/T-anti-palindromic matrix pencil we have the following relations

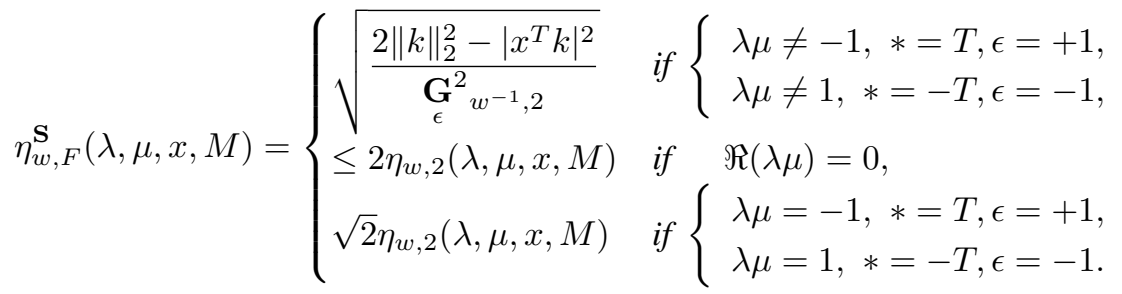

Consider the matrix polynomial of the form $M \otimes f(\lambda)=\sum_{j=0}^{m} \lambda^{j} M_{j}$. A special case of Theorem 4.1 can be derived for non-homogeneous matrix polynomials. We get the following results:

THEOREM 4.3. Let $M \in M_{m+1}\left(\mathbb{C}^{n \times n}\right)$ be a T-palindromic/T-anti-palindromic matrix polynomial of the form (4.1) with $\lambda=1$. Let $k=-M(\mu) x, Z_{M_{j}}^{\epsilon}$ given in equation (3.1), and let $P_{x}=I-x x^{H}$. Then we have

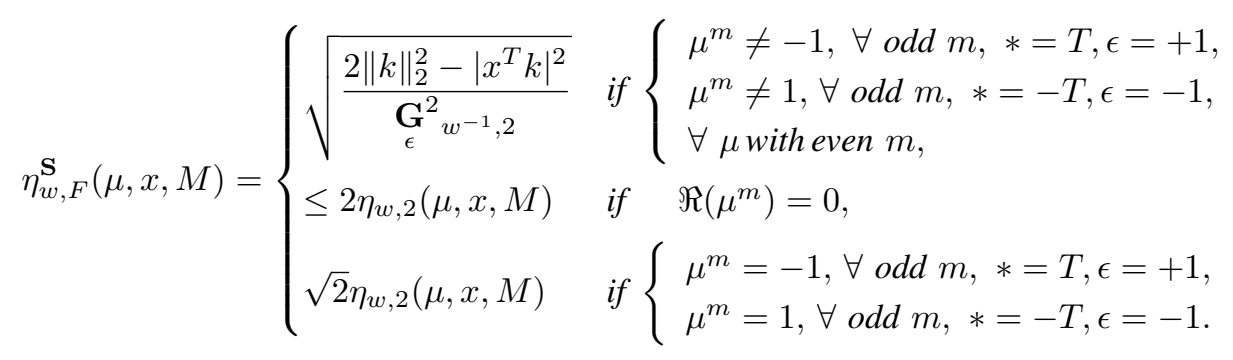

with the perturbation matrices

$$
\Delta M_{j}:=\left\{\begin{aligned}
& \bar{Z}_{M_{j}}^{\epsilon} \bar{x} x^{T} k x^{H}+\epsilon \bar{Z}_{M_{m-j}}^{\epsilon} \bar{x} k^{T} P_{x}+ \bar{Z}_{M_{j}}^{\epsilon} P_{x}^{T} k x^{H} \\
& \text { if }\left\{\begin{array}{l}
\mu^{m} \neq-1, \forall \text { odd } m, *=T, \epsilon=+1, \\
\mu^{m} \neq 1, \forall \text { odd } m, *=-T, \epsilon=-1, \\
\forall \mu \text { with even } m,
\end{array}\right. \\
& \pm Z_{M_{m-j}}^{\epsilon} \bar{x} k^{T} P_{x}+Z_{M_{j}}^{\epsilon} \bar{x} P_{x}^{T} k x^{H} \\
& \text { if }\left\{\begin{array}{l}
\mu^{m}=-1, \forall \text { odd } m, *=T, \epsilon=+1, \\
\mu^{m}=1, \forall \text { odd } m, *=-T, \epsilon=-1 .
\end{array}\right.
\end{aligned}\right.
$$


Proof. The proof follows by substituting $f_{j}=\lambda^{j}$ in Theorem 3.1.

COROLLARY 4.4. For a matrix pencil we have the following relations

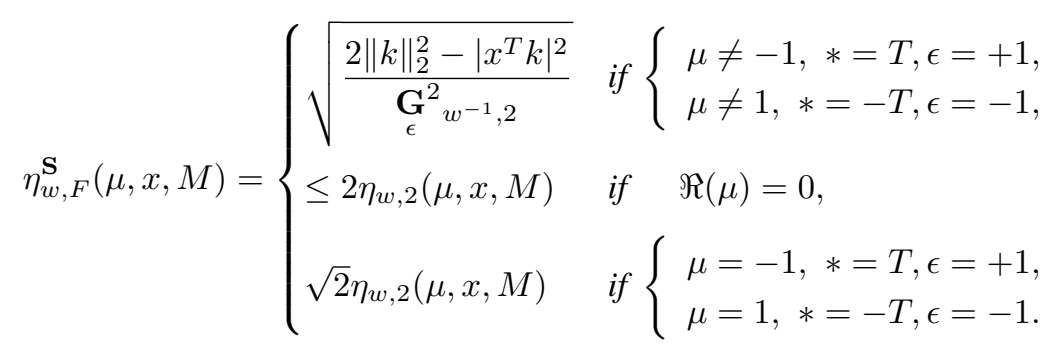

Proof. The proof follows from the previous theorem.

Now we can discuss a simpler case. Consider the matrix polynomial of the form (4.1). We derive results similar to Theorem 3.2.

THEOREM 4.5. Let $\mathbf{L} \in M_{m+1}\left(\mathbb{C}^{n \times n}\right)$ be a $H$-palindromic/H-anti-palindromic matrix polynomial. Let $k=-M(\lambda, \mu) x, Z_{M_{j}}^{\epsilon}$ be given in equation (3.1), and $P_{x}=I-x x^{H}$. Then we have

$$
\eta_{w, F}^{\mathbf{S}}(\lambda, \mu, x, M)=\left\{\begin{array}{l}
\sqrt{\frac{2\|k\|_{2}^{2}-\left|x^{H}\right|^{2}}{\mathbf{G}_{\epsilon}^{2} w^{-1}, 2}} \quad \text { if }\left\{\begin{array}{l}
\lambda^{m} \mu^{m} \neq-1, \forall \text { odd } m, *=H, \epsilon=+1, \\
\lambda^{m} \mu^{m} \neq 1, \forall \text { odd } m, *=H, \epsilon=-1, \\
\forall(\lambda, \mu) \text { with even } m,
\end{array}\right. \\
\sqrt{2} \eta_{w, 2}(\lambda, \mu, x, M) \\
\leq 2 \eta_{w, 2}(\lambda, \mu, x, M) \quad \text { if }\left\{\begin{array}{l}
\lambda^{m} \mu^{m}=-1, \text { m-odd }, *=H, \epsilon=+1, \\
\lambda^{m} \mu^{m}=1, \text { modd } *=H, \epsilon=-1,
\end{array}\right.
\end{array}\right.
$$

with the perturbation matrices

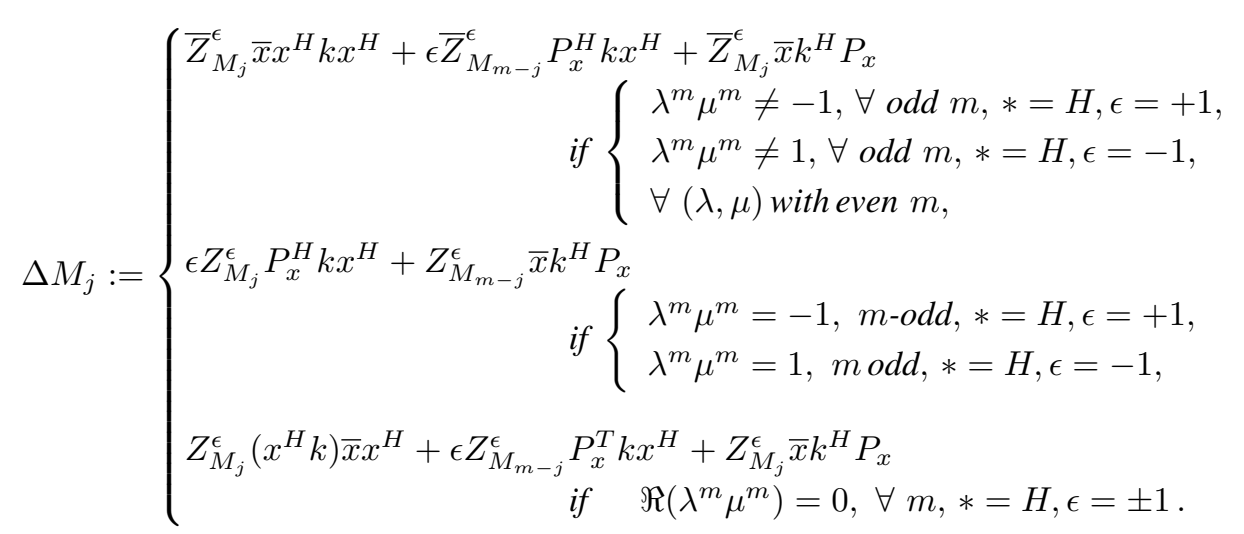

Proof. The proof follows from the previous theorem by substituting $f_{j}=\lambda^{j} \mu^{m-j}$. When $m$ is odd, we have the following two cases:

Case $A:(\lambda, \mu)=(-1,1),(1,-1)$ for an $H$-palindromic structure.

Case $B:(\lambda, \mu)=(1,1),(-1,-1)$ for an $H$-anti-palindromic structure.

In all the two cases we have $x^{H} k=0$, when $m$ is odd. Hence the minimum-norm solution of $\sum_{j=0}^{m} f_{j} a_{j j}=x^{H} k$ is $a_{j j}=0$. Similarly, we have $\sum_{j=0}^{m} f_{j} b_{j}=U_{1}^{H} k$. Then we have for 
$j=0,1, \ldots, m:$

$$
\begin{aligned}
\Delta M_{j} & =\bar{U}\left[\begin{array}{cc}
0 & \epsilon Z_{M_{m-j}}^{\epsilon}\left(U_{1}^{H} k\right)^{H} \\
Z_{M_{j}}^{\epsilon} U_{1}^{H} k & D_{j, j}
\end{array}\right] U^{H} \quad \text { and } \\
\Delta M_{j} & =\epsilon Z_{M_{m-j}}^{\epsilon} \bar{x} x^{H} P_{x}+Z_{M_{j}}^{\epsilon} P_{x}^{H} k x^{H}
\end{aligned}
$$

and the backward error is given by

$$
\eta_{w, F}^{\mathbf{S}}(\lambda, \mu, x, M)=\sqrt{2} \frac{\|k\|}{H_{w^{-1}, 2}}=\sqrt{2} \eta_{w, 2}(\lambda, \mu, x, M) .
$$

We see that the backward error of $M$ for homogeneous matrix polynomials with $H$-palindromic and $H$-anti-palindromic structures are exactly equal to $\sqrt{2}$ times the unstructured backward error when an approximate eigenpair becomes an exact eigenpair of a suitable minimal perturbed polynomial.

REMARK 4.6. Considering the matrix polynomial of the form $M \otimes f(\lambda)=\sum_{j=0}^{m} \lambda^{j} M_{j}$, the special cases of Theorem 4.5 can be derived for the case of non-homogeneous matrix polynomials.

4.1. The relation between $\boldsymbol{G}_{\boldsymbol{w}^{-1,2}}$ and $\boldsymbol{H}_{\boldsymbol{w}^{-1,2}}$. Consider the matrix polynomial $M(c, s)=\sum_{j=0}^{m} c^{j} s^{m-j} M_{j}$. Let $\tilde{m}=m / 2+1$ when $\mathrm{m}$ is even and $\tilde{m}=(m+1) / 2$ when $\mathrm{m}$ is odd. Then consider

$$
\mathbf{G}_{\epsilon, 2}(y)=\left\|\left(\alpha_{0} y_{0}, \alpha_{1} y_{1}, \ldots, \alpha_{(\tilde{m}-1)} y_{(\tilde{m}-1)}\right)\right\|_{\alpha, 2}
$$

where $w_{j}=w_{m-j}, y_{j}=f_{j}+\epsilon f_{m-j}, j=0,1,2, \ldots, \tilde{m}-1$, and define the functions $\alpha_{j} f_{j}=w_{j} f_{j}+\epsilon w_{m-j} f_{m-j}$. Note that when $m$ is even, we define $\alpha_{m / 2}=w_{m / 2}$ and $y_{m / 2}=f_{m / 2}$, where $f_{j}=\lambda^{j} \mu^{m-j}$. For a $T$-palindromic/H-palindromic structure, the structured backward error is half as large as the unstructured backward error for all $m$ when $\Re\left(f_{j} \bar{f}_{m-j}\right) \geq 0$, and similarly, for a $T$-palindromic/H-palindromic structure, the structured backward error is two times larger than the unstructured backward error for all $m$ when $\Re\left(f_{j} \bar{f}_{m-j}\right) \leq 0$.

COROLlARY 4.7. Let $M \in M_{m+1}\left(\mathbb{C}^{n \times n}\right)$ be a T-palindromic/H-palindromic matrix polynomial of the form (1.1). Let $(\lambda, \mu) \neq(1,-1),(-1,1)$, and let $\epsilon=+1$.

- If $\Re\left(f_{j} \bar{f}_{m-j}\right) \geq 0$, then $\mathbf{G}_{w^{-1}, 2} \geq \frac{1}{\sqrt{2}} H_{w^{-1}, 2}$ and $\eta_{w, F}^{\mathbf{S}}(\lambda, \mu, x, M) \leq 2 \eta_{w, 2}(\lambda, \mu, x, M)$.

- If $\Re\left(f_{j} \bar{f}_{m-j}\right) \leq 0$, then $\mathbf{G}_{w^{-1}, 2} \leq \frac{1}{\sqrt{2}} H_{w^{-1}, 2}$ and $\eta_{w, F}^{\mathbf{S}}(\lambda, \mu, x, M) \geq 2 \eta_{w, 2}(\lambda, \mu, x, M)$.

Proof. The proof follows from Theorem 3.1 and Theorem 3.2.

For T-anti-palindromic/H-anti-palindromic structures, the structured backward error is half as large as the unstructured backward error for all $m$ when $\Re\left(f_{j} \bar{f}_{m-j}\right) \leq 0$, and similarly, for T-anti-palindromic/H-anti-palindromic structures, the structured backward error is two times larger than the unstructured backward error for all $m$ when $\Re\left(f_{j} \bar{f}_{m-j}\right) \geq 0$.

COROLlARY 4.8. Let $M \in M_{m+1}\left(\mathbb{C}^{n \times n}\right)$ be a T-anti-palindromic/H-anti-palindromic matrix polynomial of the form (4.1). Let $m$ be odd with $(\lambda, \mu) \neq(1,1),(-1,-1)$, and let $\epsilon=-1$. Then we have the following:

- If $\Re\left(f_{j} \bar{f}_{m-j}\right) \leq 0$,

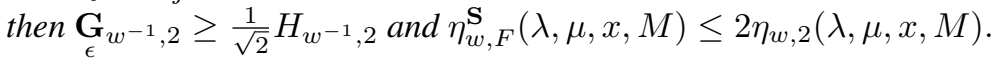

- If $\Re\left(f_{j} \bar{f}_{m-j}\right) \geq 0$, then $\mathbf{G}_{\epsilon} w^{-1}, 2 \leq \frac{1}{\sqrt{2}} H_{w^{-1}, 2}$ and $\eta_{w, F}^{\mathbf{S}}(\lambda, \mu, x, M) \geq 2 \eta_{w, 2}(\lambda, \mu, x, M)$.

Proof. The proof follows from Theorem 3.1 and Theorem 3.2. 
TABLE 4.2

For a palindromic structure, where $\epsilon=+1$.

\begin{tabular}{l|l|l|l}
$m$ & $(\lambda, \mu)$ & $\Re\left(f_{j} \bar{f}_{m-j}\right)$ & $*=\{T, H\}, \epsilon=+1$ \\
\hline odd & $\neq(1,-1),(-1,1)$ & $\geq 0$ & $\Lambda_{\epsilon, F}^{\mathbf{S}}(M) \subseteq \Lambda_{2 \epsilon, 2}(M)$ \\
\hline odd & $\neq(1,-1),(-1,1)$ & $\leq 0$ & $\Lambda_{2 \epsilon, 2}(M) \subseteq \Lambda_{\epsilon, F}^{\mathbf{S}}(M)$ \\
\hline even & $\forall(\lambda, \mu)$ & $\geq 0$ & $\Lambda_{\epsilon, F}^{\mathbf{S}}(M) \subseteq \Lambda_{2 \epsilon, 2}(M)$ \\
\hline even & $\forall(\lambda, \mu)$ & $\leq 0$ & $\Lambda_{2 \epsilon, 2}(M) \subseteq \Lambda_{\epsilon, F}^{\mathbf{S}}(M)$
\end{tabular}

TABLE 4.3

For an anti-palindromic structure, where $\epsilon=-1$.

\begin{tabular}{l|l|l|l}
$m$ & $(\lambda, \mu)$ & $\Re\left(f_{j} \bar{f}_{m-j}\right)$ & $*=\{T, H\}, \epsilon=-1$ \\
\hline odd & $\neq(1,1),(-1,-1)$ & $\leq 0$ & $\Lambda_{\epsilon, F}^{\mathbf{S}}(M) \subseteq \Lambda_{2 \epsilon, 2}(M)$ \\
\hline odd & $\neq(1,1),(-1,-1)$ & $\geq 0$ & $\Lambda_{2 \epsilon, 2}(M) \subseteq \Lambda_{\epsilon, F}^{\mathbf{S}}(M)$ \\
\hline even & $\forall(\lambda, \mu)$ & $\leq 0$ & $\Lambda_{\epsilon, F}^{\mathbf{S}}(M) \subseteq \Lambda_{2 \epsilon, 2}(M)$ \\
\hline even & $\forall(\lambda, \mu)$ & $\geq 0$ & $\Lambda_{2 \epsilon, 2}(M) \subseteq \Lambda_{\epsilon, F}^{\mathbf{S}}(M)$
\end{tabular}

5. Structured versus unstructured pseudospectra. Let $M \otimes f=\sum_{j=0}^{m} f_{j} M_{j}$, $f=\left[f_{0}, f_{1}, \ldots, f_{m}\right] \in \mathbb{C}^{m+1}, M=\left[M_{0}, M_{1}, \ldots, M_{m}\right] \in\left(\mathbb{C}^{n \times n}\right)^{m+1}$. Then we define the pseudospectra and structured pseudospectra of $M \otimes f$ as follows for the Frobenius norm of a matrix:

$$
\begin{aligned}
& \Lambda_{\epsilon, w}(M):=\left\{f \in \mathbb{C}^{m+1}: \operatorname{det}((M+\Delta M) \otimes f)=0, \Delta M \in M_{m+1}\left(\mathbb{C}^{n \times n}\right)\right\}, \\
& \Lambda_{\epsilon, w}^{\mathbf{S}}(M):=\left\{f \in \mathbb{C}^{m+1}: \operatorname{det}((M+\Delta M) \otimes f)=0, \Delta M \in M_{m+1}^{\mathbf{S}}\left(\mathbb{C}^{n \times n}\right)\right\} .
\end{aligned}
$$

Proposition 5.1. Let $M \in M_{m+1}\left(\mathbb{C}^{n \times n}\right)$ be of the form $M \otimes f=\sum_{j=0}^{m} f_{j} M_{j}$. Then

$$
\begin{aligned}
& \Lambda_{\epsilon, w}(M)=\left\{f \in \mathbb{C}^{m+1}: \Delta M \in \mathbf{S}: \eta_{w, l}^{\mathbf{S}}(f, x, M) \leq \epsilon\right\}, \quad l=\{2, F\}, \\
& \Lambda_{\epsilon, w}^{\mathbf{S}}(M)=\left\{f \in \mathbb{C}^{m+1}: \Delta M \in M_{m+1}\left(\mathbb{C}^{n \times n}\right), \eta_{w, l}(f, x, M) \leq \epsilon\right\}, \quad l=\{2, F\} .
\end{aligned}
$$

Substituting $f_{j}=c^{j} s^{m-j}$ in Proposition 5.1, we get the desired result for homogeneous matrix polynomials. For T-palindromic/anti-palindromic structures, the $\epsilon$-pseudospectrum of $M$ in the Frobenius norm is $\sqrt{2}$ times the pseudospectrum of $M$ in the spectral norm when $w=(1,1, \ldots, 1)$ and $(\lambda, \mu)=(1, \mp 1)$.

COROLlary 5.2. Let $M \in M_{m+1}\left(\mathbb{C}^{n \times n}\right)$ be a T-palindromic/T-anti-palindromic matrix polynomial $M(c, s)=\sum_{j=0}^{m} c^{j} s^{m-j} M_{j}$. For $(\lambda, \mu)=(1, \mp 1)$ and when $m$ is odd, we have

$$
\Lambda_{\epsilon, F}^{\mathbf{S}}(M)=\Lambda_{\sqrt{2} \epsilon, 2}(M) .
$$

Proof. The proof follows from Theorem 4.1.

Corollary 5.3. Let $M \in M_{m+1}\left(\mathbb{C}^{n \times n}\right)$ be a T-palindromic/T-anti-palindromic matrix polynomial $M(c, s)=\sum_{j=0}^{m} c^{j} s^{m-j} M_{j}$. For $(\lambda, \mu)=(1,1),(\lambda, \mu)=(-1,-1)$ with m-even, we have

$$
\Lambda_{\epsilon, F}^{\mathbf{S}}(M) \subset \Lambda_{\sqrt{2} \epsilon, 2}(M)
$$


Proof. The proof follows from Theorem 4.1.

Corollary 5.4. Let $M \in M_{m+1}\left(\mathbb{C}^{n \times n}\right)$ be a T-palindromic/T-anti-palindromic matrix polynomial of the form $M(c, s)=\sum_{j=0}^{m} c^{j} s^{m-j} M_{j}$. Let $(\lambda, \mu)=(1, \mp 1)$. Then for even $m$, we get

$$
\Lambda_{\epsilon, F}^{\mathrm{S}}(M) \subset \Lambda_{\sqrt{2} \epsilon, 2}(M)
$$

Proof. The proof follows from Theorem 4.1.

Corollary 5.5. Let $M \in M_{m+1}\left(\mathbb{C}^{n \times n}\right)$ be a T-palindromic/T-anti-palindromic matrix polynomial of the form $M(c, s)=\sum_{j=0}^{m} c^{j} s^{m-j} M_{j}$. Let $(\lambda, \mu) \neq(1, \mp 1),(\lambda, \mu) \neq$ $(1, \pm 1) \forall m$. Then

(a) for a T-palindromic structure, we have $\Lambda_{\epsilon, F}^{\mathrm{S}}(M) \subset \Lambda_{\sqrt{2} \epsilon, 2}(M)$.

(b) For a T-anti-palindromic structure, we have $\Lambda_{\sqrt{2} \epsilon, 2}(M) \subset \Lambda_{\epsilon, F}^{\mathbf{S}}(M)$.

Proof. The proof follows from Theorem 4.1.

EXAMPLE 5.6. Consider the rational eigenvalue problem in homogeneous form

$$
\left(s M_{0}+c M_{1}+\frac{c s}{\sigma_{1} s-c} M_{2}\right) y=0, \quad \sigma_{1}=12
$$

where $(c, s) \backslash\{0\}$. Let $f=\left[f_{0}, f_{1}, f_{2}\right]^{T}$ such that $f_{0}=s, f_{1}=c, f_{2}=\frac{c s}{\sigma_{1} s-c}$. Then (5.1) becomes $(M \otimes f) y=0$, where $M=\left[M_{0}, M_{1}, M_{2}\right] \in\left(\mathbb{C}^{n \times n}\right)^{3}$.

(a) Let the coefficient matrices of (5.1) have a $T$-palindromic structure, with $M_{0}=M_{2}^{T}$, $M_{1}=M_{1}^{T}$, where

$$
\begin{aligned}
M_{0} & =\left[\begin{array}{ccc}
1 & 2 & 3 \\
2+i & 2+2 i & 7+i \\
3 & 2+i & 6-3 i
\end{array}\right], \quad M_{1}=\left[\begin{array}{ccc}
1 & 1-i & 1+i \\
1-i & 2+2 i & 2+i \\
1+i & 2+i & 6-3 i
\end{array}\right], \\
M_{2} & =\left[\begin{array}{ccc}
1 & 2+i & 3 \\
2 & 2+2 i & 2+i \\
3 & 7+i & 6-3 i
\end{array}\right] .
\end{aligned}
$$

We choose here $x=\left[\begin{array}{lll}i / \sqrt{3} & -i / \sqrt{3} & 1 / \sqrt{3}\end{array}\right]^{T}$ as an approximate eigenvector corresponding to an approximate eigenvalue $(\lambda, \mu) \in \mathbb{C}^{2} \backslash\{(0,0)\}$ of $M \otimes f$ such that $(M \otimes \psi) x \approx 0$, where $\psi:=\left[\psi_{0}, \psi_{1}, \psi_{2}\right]^{T}, \psi_{0}=\mu, \psi_{1}=\lambda, \psi_{2}=\frac{\lambda \mu}{\sigma_{1} \mu-\lambda}$. Then there exists a minimalnorm perturbation $\Delta M:=\left[\Delta M_{0}, \Delta M_{1}, \Delta M_{2}\right] \in\left(\mathbb{C}^{3 \times 3}\right)^{3}$ in the Frobenius norm such that $((M+\Delta M) \otimes \psi) x=0$, and the corresponding backward errors for different values of $(\lambda, \mu) \in \mathbb{C}^{2} \backslash\{(0,0)\}$ are given by

\begin{tabular}{l|l|l|l}
$(\lambda, \mu)$ & $\mathbf{S}$ & $\eta_{2}(\psi, x, M)$ & $\eta_{F}^{\mathbf{S}}(\psi, x, M)$ \\
\hline$(1,2)$ & $T$-palindromic & 8.1604 & 10.9467 \\
\hline$(3,5)$ & $T$-palindromic & 7.9795 & 10.6687 \\
\hline$(i, 1+5 i)$ & $T$-palindromic & 7.4073 & 10.0112
\end{tabular}


(b) Let the coefficient matrices of (5.1) be $H$-palindromic with $M_{0}=M_{2}^{H}, M_{1}=M_{1}^{H}$, where

$$
\begin{aligned}
M_{0} & =\left[\begin{array}{ccc}
1 & 2 & 3 \\
2+i & 2+2 i & 7+i \\
3 & 2+i & 6-3 i
\end{array}\right], \quad M_{1}=\left[\begin{array}{ccc}
1 & 1-i & 1+i \\
1+i & 2 & 2+i \\
1-i & 2-i & 6
\end{array}\right], \\
M_{2} & =\left[\begin{array}{ccc}
1 & 2-i & 3 \\
2 & 2-2 i & 2-i \\
3 & 7-i & 6+3 i
\end{array}\right] .
\end{aligned}
$$

Let $x=\left[\begin{array}{lll}i / \sqrt{3} & -i / \sqrt{3} & 1 / \sqrt{3}\end{array}\right]^{T}$ be an approximate eigenvector corresponding to an approximate eigenvalue $(\lambda, \mu) \in \mathbb{C}^{2} \backslash\{(0,0)\}$ of $M \otimes f$ that is $\left.M \otimes \psi\right) x \approx 0$. Then there exists $\Delta M=\left[\Delta M_{0}, \Delta M_{1}, \Delta M_{2}\right] \in\left(\mathbb{C}^{3 \times 3}\right)^{3}$ such that $((M+\Delta M) \otimes \psi) x=0$, and the corresponding backward errors for different values of $(\lambda, \mu)$ are as follows:

\begin{tabular}{l|l|l|l}
$(\lambda, \mu)$ & $\mathbf{S}$ & $\eta_{2}(\psi, x, M)$ & $\eta_{F}^{\mathbf{S}}(\psi, x, M)$ \\
\hline$(i, 2-i)$ & $H$-palindromic & 5.5175 & 83.4494 \\
\hline$(3 i, 5-4 i)$ & $H$-palindromic & 5.2493 & 87.7947
\end{tabular}

6. Conclusion. In this work, we have extended the construction of structured backward errors for a polynomial eigenvalue problem to general nonlinear eigenvalue problems. A systematic framework for the construction of appropriately structured backward errors is discussed for the classes of T-palindromic, T-anti-palindromic, $\mathrm{H}$-palindromic, and $\mathrm{H}$ anti-palindromic problems. The resulting minimal perturbation is unique in the case of the Frobenius norm and has infinitely many solutions for the matrix 2-norm. Using these results, we have determined structured pseudospectra and have compared these results with the backward errors of the unstructured backward errors of the nonlinear eigenvalue problem. The results are similar to the previous work in this direction $[1,2]$ but for different structures that have not been discussed before. The relations between structured and unstructured backward errors and pseudospectra have been derived. This work can be extended to more than one specified eigenpairs, which will be discussed in our future research work.

\section{REFERENCES}

[1] B. AdhiKari, Backward Perturbation and Sensitivity Analysis of Structured Polynomial Eigenvalue Problem, $\mathrm{PhD}$. Thesis, Dept. of Mathematics, IIT Guwahati, Guwahati, 2008.

[2] - Backward errors and linearizations for palindromic matrix polynomials, Preprint on arXiv, 2009. https://arxiv.org/abs/0812.4154.

[3] B. AdhiKari AND R. Alam, Structured backward errors and pseudospectra of structured matrix pencils, SIAM J. Matrix Anal. Appl., 31 (2009), pp. 331-359.

[4] S. S. AHMAD, Pseudospectra of matrix pencils and their applications in the perturbation analysis of eigenvalues and eigendecompositions. PhD. Thesis, Dept. of Mathematics, IIT Guwahati, Guwahati, 2007.

[5] S. S. Ahmad AND R. Alam, Pseudospectra, critical points and multiple eigenvalues of matrix polynomials, Linear Algebra Appl., 430 (2009), pp. 1171-1195.

[6] S. S. AHMAD AND V. MeHRMANN, Backward errors and pseudospectra for structured nonlinear eigenvalue problems, Oper. Matrices, 10 (2016), pp. 539-556.

[7] - Perturbation analysis for complex symmetric, skew symmetric, even and odd matrix polynomials, Electron. Trans. Num. Anal., 38 (2011), pp. 275-302. http://etna.ricam.oeaw.ac.at/vol.38.2011/pp275-302.dir/pp275-302.pdf

[8] - Backward errors for eigenvalues and eigenvectors of Hermitian, skew-Hermitian, H-even and H-odd matrix polynomials, Linear Multilinear Algebra, 61 (2013), pp. 1244-1266.

[9] I. Gohberg, P. LANCASTER, AND L. Rodman, Spectral analysis of self adjoint matrix polynomials, Ann. of Math., 112 (1980), pp. 33-71. 
[10] N. J. Higham, D. S. Mackey, N. Mackey, And F. Tisseur, Symmetric linearizations for matrix polynomials, SIAM J. Matrix Anal. Appl., 29 (2006), pp. 143-159.

[11] N. J. Higham, D. S. MACKEY, AND F. TISSEUR. The conditioning of linearizations of matrix polynomials, SIAM J. Matrix Anal. Appl., 28 (2006), pp. 1005-1028.

[12] H. Y. HU AND Z. H. WANG, Dynamics of Controlled Mechanical Systems with Delayed Feedback, Springer, Berlin, 2002.

[13] E. JARlebring, Critical delays and polynomial eigenvalue problems, J. Comput. Appl. Math., 224 (2009), pp. 296-306.

[14] E. JARLEBRING AND T. DAMM, The Lambert $W$ function and the spectrum of some multidimensional time-delay systems, Automatica J. IFAC, 43 (2007), pp. 2124-2128.

[15] R. C. LI, W. W. LIN, AND C. S. WANG, Structured backward error for palindromic polynomial eigenvalue problems, Numer. Math., 116 (2010), pp. 95-122.

[16] D. S. Mackey, N. Mackey, C. Mehl, and V. Mehrmann, Structured polynomial eigenvalue problems: Good vibrations from good linearizations, SIAM J. Matrix Anal. Appl., 28 (2006), pp. 1029-1051.

[17] — Vector spaces of linearizations for matrix polynomials, SIAM J. Matrix Anal. Appl., 28 (2006), pp. 971-1004. 